\title{
V017 SEISMIC RESERVOIR MONITORING IN THE ARABIAN GULF
}

PANOS G. KELAMIS', RICHARD D. CHIMBLO ${ }^{1}$, ASAAD A. AL-TOWAILIB ${ }^{2}$, DIA S. AL-QUDAIHY², RICHARD C. UDEN ${ }^{3}$ and CRAIG S. MARSHALL 1 Saudi Aramco, Geophysical R\&D, PO Box 8073, Dhahran 31311, Saudi Arabia

${ }^{2}$ Saudi Aramco, Reservoir Engineering

${ }^{3}$ Scientific Software-Intercomp UK Ltd

\section{Introduction}

Seismic reservoir monitoring is gaining popularity within the Exploration and Producing industry. Analysis of repetitive seismic surveys provides direct measurements of the fluid movements and saturation changes within the hydrocarbon accumulation. Tracking saturation fronts is essential to design and improve depletion and production strategies in order to properly manage as well as predict the performance of the field. In this paper, we present the analysis and results of a seismic reservoir monitoring project over an offshore clastic field in the Arabian Gulf. After rotation and repositioning of the two seismic datasets, a difference 3D volume was computed, validated by geological data and calibrated to a reservoir simulation model that has been history matched. The seismic difference between the two 3D surveys indicates gas encroachment within the upper part of the reservoir and is in overall agreement with production logging measurements at the wells.

\section{Methodology}

In 1992 Saudi Aramco acquired a 3D seismic dataset over an offshore clastic reservoir. A 3D dataset had already been acquired in 1979 covering a sector area of the same Field. The 1979 and 1992 3D surveys cover a common area of approximately 62 square kilometers. The main producing sandstone reservoir in this Field lies within the Khafji member of the lower Cretaceous Wasia formation. The main sand consists of channelized and sheet sand bodies that were deposited in a fluvial dominated delta system that prograded over a pre-existing shallow marine carbonate platform. During a production period of more than twenty years, measurements at wells have indicated gas encroachment within the main sand body. Analyzing the repetitive 3D seismics, the main objective was to detect any seismic differences, if any, and how they are related to the movement of the gas/oil saturation front between 1979 and 1992.

The difference in acquisition for the 1979 and 1992 datasets (one streamer versus four, differences in source, offset, bin size, line spacing, orientation, etc.) posed an early problem. It was decided to process the two surveys in the direction of their main orientations taking into account a common maximum offset. The processing flow emphasized relative amplitude for both datasets. Then, an after stack seismic registration and calibration was performed in three steps :

a) Rotation of the 1979 data to the orientation of the 1992 - this is recommended rather than bin one or other of the datasets in the other's orientation.

b) Equalization between the two datasets to remove any prevailing residual wavelet differences.

c) Repositioning the 1979 survey data to the 1992 survey orientation using cross-correlations. 
There was a considerable number of iterations between stages (b) and (c) in order to minimize the seismic difference above and below the reservoir. There were few changes outside the reservoir zone and these were attributed to permanent changes between the two surveys. The final seismic difference, after registration and calibration, revealed a well-defined signature at the upper part of the reservoir. By integrating geological and engineering data as well as results from simulation, it was found that changes in the gas saturation are related to changes in acoustic impedance. Therefore, the seismic difference which essentially represents acoustic impedance changes, can be considered a direct measurement of the gas saturation front. Further analysis involving production data from 1979 and 1992 indicated gas encroachment within the upper sands. The gas fronts were well correlated with the seismic difference signature.

\section{Conclusions}

The seismic reservoir monitoring project in the Arabian Gulf has demonstrated the potential of 4D seismic technology as a tool to track fluid movements within the reservoir and, in particular, in regions with minimum well control. It was also shown that proper registration and calibration of the repetitive seismic datasets is a very important and critical process in the analysis. The integration of all available data (geological, engineering, production etc.) as well as calibration to a reference simulation model remains the key in seismic reservoir monitoring.

\section{Acknowledgements}

We express our thanks to the Saudi Arabian Oil Company (Saudi Aramco) for encouraging this work and for granting permission to present this paper. Discussions with Yahya Shinawi, Mike Chichakli and Mike. Strimenos from the Reservoir Engineering Department of Saudi Aramco were appreciated. We also thank the Geophysical Processing Division of Saudi Aramco and especially Jeff Hirsch for processing the 3D seismic data. 